\title{
Solvability of multi-point boundary value problems on the half-line
}

\author{
Chan-Gyun Kimª, \\ ${ }^{a}$ Department of Mathematics, Pusan National University, 609-735, Korea. \\ This paper is dedicated to Professor Ljubomir Ćirić \\ Communicated by Professor V. Berinde
}

\begin{abstract}
In this work, using the Leray-Schauder continuation principle, we study the existence of at least one solution to the quasilinear second-order multi-point boundary value problems on the half-line.
\end{abstract}

Keywords: Solvability, $m$-point boundary value problem, $p$-Laplacian, half-line 2010 MSC: Primary 34B10; Secondary 34B15, 34B40.

\section{Introduction}

Boundary value problems on the half-line arise quite naturally in the study of radially symmetric solutions of nonlinear elliptic equations and in various applications such as an unsteady flow of gas through a semiinfinite porous media, theory of drain flows and plasma physics. There have been many works concerning the existence of solutions for the boundary value problems on the half-line. We refer the reader to [1, 2, 3, 4, 6, 8, 9, 10, 11, 12, 13, 14, 15, 16, 19, 20, 22, 23] and the references therein.

Recently, Lian and Ge ([1]) studied the second-order three-point boundary value problem

$$
\begin{aligned}
& x^{\prime \prime}(t)+g\left(t, x(t), x^{\prime}(t)\right)=0, \text { a.e. } t \in \mathbb{R}_{+}, \\
& x(0)=\alpha x(\eta), \lim _{t \rightarrow \infty} x^{\prime}(t)=0,
\end{aligned}
$$

where $\mathbb{R}_{+}=[0, \infty), \alpha \neq 1$ and $\eta>0$. The authors investigated the existence of at least one solution under the assumption that $g(t, \cdot, \cdot)$ and $\operatorname{tg}(t, \cdot, \cdot)$ are Carathéodory with respect to $L^{1}\left(\mathbb{R}_{+}\right)$.

\footnotetext{
${ }^{*}$ Corresponding author

Email address: cgkim75@pusan.ac.kr (Chan-Gyun Kim )
} 
More recently, Kosmatov ([10]) studied the second-order nonlinear differential equation

$$
\left(q(t) y^{\prime}(t)\right)^{\prime}=k\left(t, y(t), y^{\prime}(t)\right) \text {, a.e. } t \in \mathbb{R}_{+},
$$

satisfying two sets of boundary conditions:

$$
y^{\prime}(0)=0, \lim _{t \rightarrow \infty} y(t)=0
$$

and

$$
y(0)=0, \quad \lim _{t \rightarrow \infty} y(t)=0,
$$

where $k: \mathbb{R}_{+} \times \mathbb{R} \times \mathbb{R} \rightarrow \mathbb{R}$ is Carathéodory with respect to $L^{1}\left(\mathbb{R}_{+}\right), \mathbb{R}=(-\infty, \infty), q \in C\left(\mathbb{R}_{+}\right) \cap$ $C^{1}(0, \infty), 1 / q \in L^{1}\left(\mathbb{R}_{+}\right)$and $q(t)>0$ for all $t \in \mathbb{R}_{+}$. The author obtained the existence of at least one solution to the above problems using the Leray-Schauder continuation principle. In the end of the paper, the author pointed out that the assumption $q(0)>0$ could be omitted, in which case one would have to work in a Banach space equipped with a weighted norm after the boundary conditions are adjusted accordingly.

Motivated by the above works ([10, 11]), we study the quasilinear second-order nonlinear differential equation

$$
\left(w(t) \varphi_{p}\left(u^{\prime}(t)\right)\right)^{\prime}+f\left(t, u(t), u^{\prime}(t)\right)=0 \text {, a.e. } t \in \mathbb{R}_{+},
$$

satisfying the following four sets of boundary conditions:

$$
\begin{gathered}
u(0)=\sum_{i=1}^{m-2} a_{i} u\left(\xi_{i}\right), \lim _{t \rightarrow \infty}\left(\varphi_{p}^{-1}(w) u^{\prime}\right)(t)=0, \\
u(0)=\sum_{i=1}^{m-2} a_{i}\left(\varphi_{p}^{-1}(w) u^{\prime}\right)\left(\xi_{i}\right), \lim _{t \rightarrow \infty}\left(\varphi_{p}^{-1}(w) u^{\prime}\right)(t)=0, \\
\lim _{t \rightarrow 0+}\left(\varphi_{p}^{-1}(w) u^{\prime}\right)(t)=0, \lim _{t \rightarrow \infty} u(t)=\sum_{i=1}^{m-2} a_{i} u\left(\xi_{i}\right), \\
\lim _{t \rightarrow 0+}\left(\varphi_{p}^{-1}(w) u^{\prime}\right)(t)=0, \lim _{t \rightarrow \infty} u(t)=\sum_{i=1}^{m-2} a_{i}\left(\varphi_{p}^{-1}(w) u^{\prime}\right)\left(\xi_{i}\right),
\end{gathered}
$$

where $\varphi_{p}(s)=|s|^{p-2} s, p>1, \xi_{i} \in \mathbb{R}_{+}$with $0 \leq \xi_{1}<\xi_{2}<\cdots<\xi_{m-2}, a_{i} \in \mathbb{R}$ with $\sum_{i=1}^{m-2} a_{i} \neq 1, w \in$ $C\left(\mathbb{R}_{+}, \mathbb{R}\right)$ and $f: \mathbb{R}_{+} \times \mathbb{R} \times \mathbb{R} \rightarrow \mathbb{R}$ is a Carathéodory function such that $f=f(t, u, v)$ is Lebesgue measurable in $t$ for all $(u, v) \in \mathbb{R} \times \mathbb{R}$ and continuous in $(u, v)$ for almost all $t \in \mathbb{R}_{+}$. We further assume the following conditions hold.

(F) There exist measurable functions $\alpha, \beta$ and $\gamma$ such that

$$
\alpha, \beta / w, \gamma \in L^{1}\left(\mathbb{R}_{+}\right)
$$

and

$$
|f(t, u, v)| \leq \alpha(t)|u|^{p-1}+\beta(t)|v|^{p-1}+\gamma(t) \text {, a.e. } t \in \mathbb{R}_{+} .
$$

(W) $\varphi_{p}^{-1}(1 / w) \in L^{1}\left(\mathbb{R}_{+}\right)$and $Z_{w}=\left\{t \in \mathbb{R}_{+} \mid w(t)=0\right\}$ is a finite set.

By a solution to problem $(P),\left(B C_{i}\right)$, we understand a function $u \in C\left(\mathbb{R}_{+}\right) \cap C^{1}\left(\mathbb{R}_{+} \backslash Z_{w}\right)$ with $w \varphi_{p}\left(u^{\prime}\right) \in$ $A C\left(\mathbb{R}_{+}\right)$satisfying $(P),\left(B C_{i}\right)(i=1,2,3,4)$.

To the author's knowledge, the multi-point boundary value problems with sign-changing weight $w$ have not been investigated until now. The purpose of this paper is to establish the existence of at least one solution to $p$-Laplacian boundary value problems $(P),\left(B C_{i}\right)(i=1,2,3,4)$ with sign-changing weight $w$. 
Since $\mathbb{R}_{+}$is not compact, the related compactness principle on a bounded interval $[0,1]$ does not hold. In addition, solutions $u$ of $(P),\left(B C_{i}\right)(i=1,2,3,4)$ may not be in $C^{1}\left(\mathbb{R}_{+}\right)$since $w$ may have zeros in $\mathbb{R}_{+}$. In order to overcome these difficulties, a new Banach space equipped with a weighted norm is introduced, and then we can proceed with the Leray-Schauder continuation principle which was used in many works (see, e.g., [5, 17, 9, 10, 11, 17]) in order to prove the existence of a solution for the problems $(P),\left(B C_{i}\right)(i=1,2,3,4)$.

The rest of this paper is organized as follows. In Section 2, a weighted Banach space and corresponding operators to problems $(P),\left(B C_{i}\right)(i=1,2,3,4)$ are introduced, and lemmas are presented. In Section 3, our main results are given, and also an example to illustrate our results is presented.

\section{Preliminaries}

Let $X$ be the Banach space

$$
X=\left\{u \in C^{1}\left(\mathbb{R}_{+} \backslash Z_{w}\right) \mid u \text { and } \varphi_{p}^{-1}(w) u^{\prime} \text { are continuous and bounded functions on } \mathbb{R}_{+}\right\}
$$

equipped with norm

$$
\|u\|=\|u\|_{\infty}+\left\|\varphi_{p}^{-1}(w) u^{\prime}\right\|_{\infty}
$$

where $\|v\|_{\infty}=\sup _{t \in \mathbb{R}_{+}}|v(t)|$ and let $Y$ be the Banach space $L^{1}\left(\mathbb{R}_{+}\right)$equipped with norm

$$
\|h\|_{1}=\int_{0}^{\infty}|h(s)| d s .
$$

For convenience, we will use the following constants

$$
\begin{aligned}
& A=1-\sum_{i=1}^{m-2} a_{i} \\
& B=|A|^{-1} \sum_{i=1}^{m-2}\left|a_{i}\right| \int_{0}^{\xi_{i}} \varphi_{p}^{-1}\left(\frac{1}{|w(s)|}\right) d s+\int_{0}^{\infty} \varphi_{p}^{-1}\left(\frac{1}{|w(s)|}\right) d s, \\
& C=\sum_{i=1}^{m-2}\left|a_{i}\right|+\int_{0}^{\infty} \varphi_{p}^{-1}\left(\frac{1}{|w(s)|}\right) d s \\
& D=|A|^{-1} \sum_{i=1}^{m-2}\left|a_{i}\right| \int_{\xi_{i}}^{\infty} \varphi_{p}^{-1}\left(\frac{1}{|w(s)|}\right) d s+\int_{0}^{\infty} \varphi_{p}^{-1}\left(\frac{1}{|w(s)|}\right) d s .
\end{aligned}
$$

For each $h \in Y$, we define, for $t \in \mathbb{R}_{+}$,

$$
\begin{aligned}
\left(T_{1} h\right)(t)= & A^{-1} \sum_{i=1}^{m-2} a_{i} \int_{0}^{\xi_{i}} \varphi_{p}^{-1}\left(\frac{1}{w(s)} \int_{s}^{\infty} h(\tau) d \tau\right) d s \\
& +\int_{0}^{t} \varphi_{p}^{-1}\left(\frac{1}{w(s)} \int_{s}^{\infty} h(\tau) d \tau\right) d s, \\
\left(T_{2} h\right)(t)= & \sum_{i=1}^{m-2} a_{i} \varphi_{p}^{-1}\left(\int_{\xi_{i}}^{\infty} h(s) d s\right) \\
& +\int_{0}^{t} \varphi_{p}^{-1}\left(\frac{1}{w(s)} \int_{s}^{\infty} h(\tau) d \tau\right) d s, \\
\left(T_{3} h\right)(t)= & A^{-1} \sum_{i=1}^{m-2} a_{i} \int_{\xi_{i}}^{\infty} \varphi_{p}^{-1}\left(\frac{1}{w(s)} \int_{0}^{s} h(\tau) d \tau\right) d s \\
& +\int_{t}^{\infty} \varphi_{p}^{-1}\left(\frac{1}{w(s)} \int_{0}^{s} h(\tau) d \tau\right) d s
\end{aligned}
$$


and

$$
\begin{aligned}
\left(T_{4} h\right)(t)= & -\sum_{i=1}^{m-2} a_{i} \varphi_{p}^{-1}\left(\int_{0}^{\xi_{i}} h(s) d s\right) \\
& +\int_{t}^{\infty} \varphi_{p}^{-1}\left(\frac{1}{w(s)} \int_{0}^{s} h(\tau) d \tau\right) d s .
\end{aligned}
$$

Then $T_{i}: Y \rightarrow X$ is well defined and for each $h \in Y, T_{i} h$ is the unique solution of the differential equation

$$
\left(w(t) \varphi_{p}\left(u^{\prime}(t)\right)\right)^{\prime}+h(t)=0 \text {, a.e. } t \in \mathbb{R}_{+},
$$

subject to the boundary conditions $\left(B C_{i}\right)(i=1,2,3,4)$.

Lemma 2.1. Let $h \in Y$. Then $T_{1} h$ satisfies

$$
\left\|T_{1} h\right\|_{\infty} \leq B\|h\|_{1}^{1 /(p-1)}
$$

and

$$
\left\|\varphi_{p}^{-1}(w)\left(T_{1} h\right)^{\prime}\right\|_{\infty} \leq\|h\|_{1}^{1 /(p-1)}
$$

Proof. Let $h \in Y$. Then, for all $t \in \mathbb{R}_{+}$, one has

$$
\begin{aligned}
\left|T_{1} h(t)\right| \leq & \left(|A|^{-1} \sum_{i=1}^{m-2}\left|a_{i}\right| \int_{0}^{\xi_{i}} \varphi_{p}^{-1}\left(\frac{1}{|w(s)|}\right) d s\right)\left(\int_{0}^{\infty}|h(s)| d s\right)^{1 /(p-1)} \\
& +\left(\int_{0}^{t} \varphi_{p}^{-1}\left(\frac{1}{|w(s)|}\right) d s\right)\left(\int_{0}^{\infty}|h(s)| d s\right)^{1 /(p-1)} \\
\leq & B\|h\|_{1}^{1 /(p-1)} .
\end{aligned}
$$

Similarly, for all $t \in \mathbb{R}_{+}$, one has

$$
\begin{aligned}
\left|\left(\varphi_{p}^{-1}(w)\left(T_{1} h\right)^{\prime}\right)(t)\right| & =\left|\varphi_{p}^{-1}\left(\int_{t}^{\infty} h(s) d s\right)\right| \\
& \leq\|h\|_{1}^{1 /(p-1)} .
\end{aligned}
$$

Thus the proof is complete.

The following lemmas can be proved by the similar manner and so we omit the proofs.

Lemma 2.2. Let $h \in Y$. Then, for each $i=2,4, T_{i} h$ satisfies

$$
\left\|T_{i} h\right\|_{\infty} \leq C\|h\|_{1}^{1 /(p-1)}
$$

and

$$
\left\|\varphi_{p}^{-1}(w)\left(T_{i} h\right)^{\prime}\right\|_{\infty} \leq\|h\|_{1}^{1 /(p-1)} .
$$

Lemma 2.3. Let $h \in Y$. Then $T_{3} h$ satisfies

$$
\left\|T_{i} h\right\|_{\infty} \leq D\|h\|_{1}^{1 /(p-1)}
$$

and

$$
\left\|\varphi_{p}^{-1}(w)\left(T_{i} h\right)^{\prime}\right\|_{\infty} \leq\|h\|_{1}^{1 /(p-1)}
$$


We define the Nemiskii operator $N: X \rightarrow Y$ by

$$
(N u)(t)=f\left(t, u(t), u^{\prime}(t)\right), t \in \mathbb{R}_{+} .
$$

It follows from $(F)$ that $N$ maps bounded sets of $X$ into bounded sets of $Y$ and is continuous. For each $i \in\{1,2,3,4\}$, define $L_{i} \triangleq T_{i} N: X \rightarrow X$. Then $L_{i}$ is well defined and problem $(P),\left(B C_{i}\right)$ has a solution $u$ if and only if $L_{i}$ has a fixed point $u$ in $X$.

To show the compactness of the operators $L_{i}(i=1,2,3,4)$, we use the following compactness criterion.

Theorem 2.4. ([2]) Let $Z$ be the space of all bounded continuous vector-valued functions on $\mathbb{R}_{+}$and $S \subset Z$. Then $S$ is relatively compact in $Z$ if the following conditions hold.

(i) $S$ is bounded in $Z$.

(ii) the functions from $S$ are equicontinuous on any compact interval of $\mathbb{R}_{+}$.

(iii) the functions from $S$ are equiconvergent, that is, given $\epsilon>0$, there exists a $T=T(\epsilon)>0$ such that $\|\phi(t)-\phi(\infty)\|_{\mathbb{R}^{n}}<\epsilon$, for all $t>T$ and all $\phi \in S$.

Lemma 2.5. For each $i \in\{1,2,3,4\}$, the mapping $L_{i}: X \rightarrow X$ is completely continuous.

Proof. We only prove that $L_{1}: X \rightarrow X$ is completely continuous since other cases can be proved by the similar manner.

First, we show that $L_{1}$ is compact. Let $\Sigma$ be bounded in $X$, i.e., there exists $M>0$ such that $\|u\| \leq M$ for all $u \in \Sigma$. Then there exists $h_{M} \in Y$ such that $|(N u)(t)| \leq h_{M}(t)$ for all $t \in \mathbb{R}_{+}$and all $u \in \Sigma$. By Lemma 2.1. $L_{1}(\Sigma)$ is bounded in $X$.

For $t_{1}, t_{2} \in \mathbb{R}_{+}$with $t_{1}<t_{2}$, one has

$$
\begin{aligned}
\left|\left(L_{1} u\right)\left(t_{1}\right)-\left(L_{1} u\right)\left(t_{2}\right)\right| & =\left|\int_{t_{1}}^{t_{2}} \varphi_{p}^{-1}\left(\frac{1}{w(s)} \int_{s}^{\infty}(N u)(\tau) d \tau\right) d s\right| \\
& \leq\left\|h_{M}\right\|_{1}^{1 /(p-1)} \int_{t_{1}}^{t_{2}} \varphi_{p}^{-1}\left(\frac{1}{|w(s)|}\right) d s
\end{aligned}
$$

and

$$
\begin{aligned}
& \left|\left(\varphi_{p}^{-1}(w)\left(L_{1} u\right)^{\prime}\right)\left(t_{1}\right)-\left(\varphi_{p}^{-1}(w)\left(L_{1} u\right)^{\prime}\right)\left(t_{2}\right)\right| \\
= & \left|\varphi_{p}^{-1}\left(\int_{t_{1}}^{\infty}(N u)(s) d s\right)-\varphi_{p}^{-1}\left(\int_{t_{2}}^{\infty}(N u)(s) d s\right)\right|,
\end{aligned}
$$

which yield that $L_{1}(\Sigma)$ and $\left\{\varphi_{p}^{-1}(w)\left(L_{1} u\right)^{\prime} \mid u \in \Sigma\right\}$ are equicontinuous on $\mathbb{R}_{+}$by the facts that $\varphi_{p}^{-1}$ is uniformly continuous on $[-1,1]$ and $|(N u)(t)| \leq h_{M}(t)$ for all $t \in \mathbb{R}_{+}$.

For $u \in \Sigma$, one has

$$
\lim _{t \rightarrow \infty}\left(\varphi_{p}^{-1}(w)\left(L_{1} u\right)^{\prime}\right)(t)=\lim _{t \rightarrow \infty} \varphi_{p}^{-1}\left(\int_{t}^{\infty}(N u)(s) d s\right)=0 .
$$

Then

$$
\begin{aligned}
\left|L_{1} u(t)-\lim _{t \rightarrow \infty} L_{1} u(t)\right| & =\left|\int_{t}^{\infty} \varphi_{p}^{-1}\left(\frac{1}{w(s)} \int_{s}^{\infty}(N u)(\tau) d \tau\right) d s\right| \\
& \leq\left\|h_{M}\right\|_{1}^{1 /(p-1)} \int_{t}^{\infty} \varphi_{p}^{-1}\left(\frac{1}{|w(s)|}\right) d s
\end{aligned}
$$

and

$$
\begin{aligned}
\left|\left(\varphi_{p}^{-1}(w)\left(L_{1} u\right)^{\prime}\right)(t)-\lim _{t \rightarrow \infty}\left(\varphi_{p}^{-1}(w)\left(L_{1} u\right)^{\prime}\right)(t)\right| & =\left|\varphi_{p}^{-1}\left(\int_{t}^{\infty}(N u)(s) d s\right)\right| \\
& \leq\left(\int_{t}^{\infty}\left|h_{M}(s)\right| d s\right)^{1 /(p-1)}
\end{aligned}
$$


which yield that $L_{1}(\Sigma)$ and $\left\{\varphi_{p}^{-1}(w)\left(L_{1} u\right)^{\prime} \mid u \in \Sigma\right\}$ are equiconvergent. By Theorem 2.4. we can conclude that $T_{1}$ is compact.

It follows from the Lebesgue dominated convergence theorem that $L_{1}: X \rightarrow X$ is continuous, and thus the proof is complete.

\section{Main results}

In this section, we give our main results.

Theorem 3.1. Assume $B^{p-1}\|\alpha\|_{1}+\|\beta / w\|_{1}<1$. Then problem $(P),\left(B C_{1}\right)$ has at least one solution for every $\gamma \in Y$.

Proof. Consider the differential equation, for $\lambda \in[0,1]$,

$$
\left(w(t) \varphi_{p}\left(u^{\prime}(t)\right)\right)^{\prime}+\lambda f\left(t, u(t), u^{\prime}(t)\right)=0 \text {, a.e. } t \in \mathbb{R}_{+},
$$

subject to the boundary condition $\left(B C_{1}\right)$.

Let $u$ be any solution of $(3.1),\left(B C_{1}\right)$. Then, by $(F)$ and Lemma 2.1, one has

$$
\begin{aligned}
\left\|\left(w \varphi_{p}\left(u^{\prime}\right)\right)^{\prime}\right\|_{1} & =\lambda\|N u\|_{1} \\
& \leq\|\alpha\|_{1}\|u\|_{\infty}^{p-1}+\|\beta / w\|_{1}\left\|\varphi_{p}^{-1}(w) u^{\prime}\right\|_{\infty}^{p-1}+\|\gamma\|_{1} \\
& \leq B^{p-1}\|\alpha\|_{1}\left\|\left(w \varphi_{p}\left(u^{\prime}\right)\right)^{\prime}\right\|_{1}+\|\beta / w\|_{1}\left\|\left(w \varphi_{p}\left(u^{\prime}\right)\right)^{\prime}\right\|_{1}+\|\gamma\|_{1},
\end{aligned}
$$

which yields

$$
\left\|\left(w \varphi_{p}\left(u^{\prime}\right)\right)^{\prime}\right\|_{1} \leq \frac{\|\gamma\|_{1}}{1-\left(B^{p-1}\|\alpha\|_{1}+\|\beta / w\|_{1}\right)} .
$$

It follows from Lemma 2.1 that the set of all possible solutions to problem (3.1), $\left(B C_{1}\right)$ is a priori bounded by a constant independent of $\lambda \in[0,1]$. Thus the proof is complete in view of the Leray-Schauder continuation principle (see, e.g., [18, 21]).

Similarly, the following results are obtained.

Theorem 3.2. Assume $C^{p-1}\|\alpha\|_{1}+\|\beta / w\|_{1}<1$. Then problems $(P),\left(B C_{i}\right)(i=2,4)$ have at least one solution for every $\gamma \in Y$.

Theorem 3.3. Assume $D^{p-1}\|\alpha\|_{1}+\|\beta / w\|_{1}<1$. Then problem $(P),\left(B C_{3}\right)$ has at least one solution for every $\gamma \in Y$.

Finally, we give an example to illustrate our results.

Example 3.4. In problems $(P),\left(B C_{i}\right)(i=1,2,3,4)$, let $p=3, m=3, a_{1}=1 / 2, \xi_{1}=1$, and

$$
w(t)=\left\{\begin{array}{l}
\varphi_{3}\left(-(1-t)^{1 / 2}\right), 0 \leq t<1 \\
\varphi_{3}\left((t-1)^{1 / 2}\right), 1 \leq t<2 \\
\varphi_{3}(\exp (t-2)), t \geq 2
\end{array}\right.
$$

Then $A=1 / 2, B=7, C=11 / 2$ and $D=8$. For any $\gamma \in Y$, we set

$$
f(t, u, v)=\frac{\sin t}{(t+70)^{2}} \varphi_{3}(u)+\frac{w(t)}{(t+70)^{2}} \varphi_{3}(v)+\gamma(t) .
$$

Then $\alpha(t)=\beta / w=1 /(t+70)^{2}$, and $\|\alpha\|_{1}=\|\beta / w\|_{1}=1 / 70$. Thus by Theorems 3.1, 3.2 and 3.3, problems $(P),\left(B C_{i}\right)(i=1,2,3,4)$ has at least one solution for every $\gamma \in Y$.

Acknowledgements: The author thanks the referee for his or her helpful remarks. 


\section{References}

[1] R.P. Agarwal, D. O'Regan, Boundary value problems of nonlinear type on the semi-infinity interval, Tohoku Math. J., 51 (1999) 391-397. 1

[2] R.P. Agarwal, D. O'Regan, "Infinite Interval Problems for Differential, Difference, and Integral Equations," Kluwer Academic, 2001. 1, 2.4

[3] S. Chen, Y. Zhang, Singular boundary value problems on a half-line, J. Math. Anal. Appl., 195 (1995) $449-468$. 1

[4] S. Djebali, O. Saifi, Positive solutions for singular BVPs with sign changing and derivative depending nonlinearity on the positive half-line, Acta Appl. Math., 110 (2010) 639-665. 1

[5] M. García-Huidobro, C.P. Gupta, R. Manásevich, A Dirichelet-Neumann $m$-point BVP with a $p$-Laplacian-like operator, Nonlinear Anal. T.M.A., 62 (2005) 1067-1089. 1

[6] Y. Guo, C. Yu, J. Wang, Existence of three positive solutions for $m$-point boundary value problems on infinite intervals, Nonlinear Anal. T.M.A., 71 (2009) 717-722. 1

[7] B. Hopkins, N. Kosmatov, Third-order boundary value problems with sign-changing solutions, Nonlinear Anal. T.M.A., 67 (2007) 126-137. 1

[8] C.G. Kim, Existence and iteration of positive solutions for multi-point boundary value problems on a half-line, Comput. Math. Appl., 61 (2011) 1898-1905. 1

[9] N. Kosmatov, Multi-point boundary value problems on an unbounded domain at resonance, Nonlinear Anal. T.M.A., 68 (2008) 2158-2171. 1, 1

[10] N. Kosmatov, Second order boundary value problems on an unbounded domain, Nonlinear Anal. T.M.A., 68 (2008) 875-882. 1. 1 ,

[11] H. Lian, W. Ge, Solvability for second-order three-point boundary value problems on a half-line, Appl. Math. Lett., 19 (2006) 1000-1006. 1, 1

[12] H. Lian, H. Pang, W. Ge, Triple positive solutions for boundary value problems on infinite intervals, Nonlinear Anal. T.M.A., 67 (2007) 2199-2207. 1

[13] S. Liang, J. Zhang, Positive solutions for singular third order boundary value problem with dependence on the first order derivative on the half-line, Acta Appl. Math., 111 (2010) 27-43. 1

[14] S. Liang, J. Zhang, Z. Wang, The existence of multiple positive solutions for multi-point boundary value problems on the half-line, J. Comput. Appl. Math., 228 (2009) 10-19. 1

[15] Y. Liu, Existence and unboundedness of positive solutions for singular boundary value problems on half-line, Appl. Math. Comput., 144 (2003) 543-556. 1

[16] R. Ma, Existence of positive solutions for second-order boundary value problems on infinite intervals, Appl. Math. Lett., 16 (2003) 33-39. 1

[17] R. Ma, D. O'Regan, Solvability of singular second order m-point boundary value problems, J. Math. Anal. Appl., 301 (2005) 124-134. 1

[18] J. Mawhin, Topological Degree Methods in Nonlinear Boundary value Problems, NSF-CBMS Regional Conf. Ser. in Math., vol. 40, Amer. Math. Soc., Providence, RI, 1979. 3

[19] D. O'Regan, B. Yan, R.P. Agarwal, Solutions in weighted spaces of singular boundary value problems on the half-line, J. Comput. Appl. Math., 205 (2007) 751-763. 1

[20] J. Xu, Z. Yang, Positive solutions for singular Sturm-Liouville boundary value problems on the half line, Electron. J. Differential Equations, 2010 (171) (2010) 1-8. 1]

[21] E. Zeidler, "Nonlinear Functional Analysis and its Applications I," Springer-Verlag, 1985, New York. 3

[22] X. Zhang, Successive iteration and positive solutions for a second-order multi-point boundary value problem on a half-line, Comput. Math. Appl., 58 (2009) 528-535. 1

[23] M. Zima, On positive solutions of boundary value problems on the half-line, J. Math. Anal. Appl., 259 (2001) 127-136. 1 